\title{
Scheduling ON-OFF home control devices
}

\author{
Catherine Plaisant \& Ben Shneiderman \\ Human-Computer Interaction Laboratory \\ Center for Automation Research \\ University of Maryland \\ College Park, MD 20742
}

\section{INTRODUCTION}

Contemporary homes include a large number of electronically controlled devices such as microwave ovens, VCRs, heating/cooling systems and security systems which can be scheduled to start and stop functioning at given times. A solution to the problem of multiple user interfaces is to provide a home automation system consisting of a single user interface and the necessary hardware to control all the devices in the home using this interface. Such a user interface must then be general enough to support scheduling any device for a variety of tasks.

The project had a highly practical orientation since this work was conducted in collaboration with a small Maryland company called Custom Command Systems. Custom Command specializes in the development and marketing of integrated entertainment, security, and automation systems for homes and commercial conference rooms. Their focus is on providing state-of-the-art systems that are easy for the home owner to use.

The scheduling interface was identified as one feature that could benefit from improved human factors design and few other studies are attempting to understand or solve the problem. It was decided to explore possible alternatives to the original interface which required a long and tedious process of incremental specification of dates, times and states through a deep menu structure.

The new scheduler had to stay compatible with the hardware environment of the home automation system: the control of the scheduler is through a touchscreen interface. The Microtouch or Elographic touchscreens that are used return a continuous flow of coordinates with a $1024 \times 1024$ resolution. This allows the dragging of objects, the identification of sliding motion and the use of a lift-off strategy for selection. This strategy reduces the error rates, allows the selection of small targets (as small as one pixel) and enables the design of interfaces that were not possible with earlier touchscreens [1]. The color, graphical screen displays are implemented under MS-DOS in EGA mode (640×350 pixels).
Users see the screen flush mounted into a wall or custom built into furniture or cabinetry. They first select the device to be scheduled and are then presented with the standard scheduling interface.

\section{DESCRIPTION OF THE PROTOTYPES}

In our designs we tried to apply direct manipulation techniques by using common representations of time (clocks, calendars, etc.) We also wanted to explore the use of high precision touchscreen capabilities. Four interfaces including a dual 12-hour clock scheduler, a 24-hour dial scheduler, a 24-hour linear scheduler and a digital scheduler were compared on a feature by feature basis [2]. Because the advantages and disadvantages of the digital scheduler are known we implemented only the three remaining designs, which are demonstrated in the videotape. User reactions were collected during an informal usability test with 14 subjects who used all three schedulers for a total duration of an hour. Subjects were mainly retired volunteers; they were given a variety of home control scheduling tasks and encouraged to comment on the interfaces [2].

With the 12-hour clock scheduler (Fig.1), users select the date by pointing at it on a familiar calendar. The time of the day is selected by rotating the hands of a 12-hour watch and a AM/PM toggle is available. Half of the screen is used for the start time and the other half for the stop time.

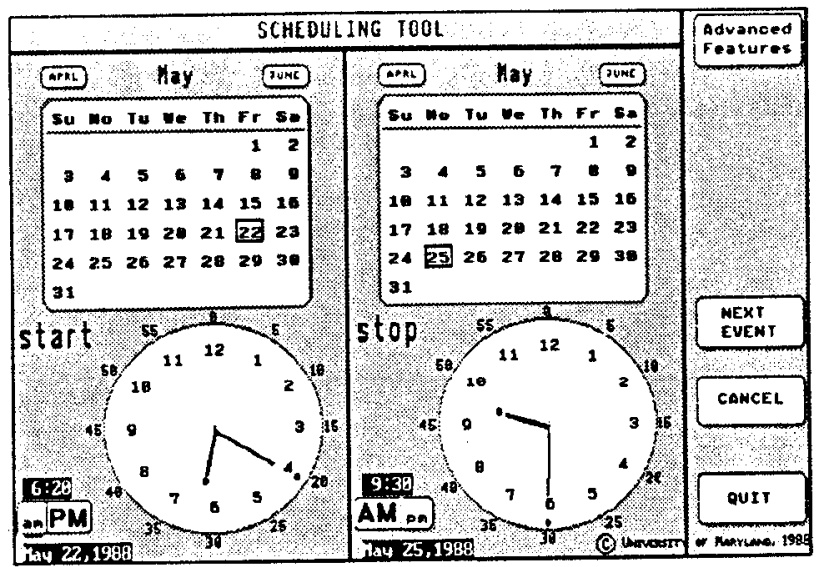

Figure 1: the dual 12-hour watch scheduler 
Contrary to our expectations the 12-hour clock was not the easiest one to use and was the only one with which errors occurred (subjects would forget the AM/PM toggle). The smooth manipulation of the clock's hands was widely appreciated but some subjects experienced difficulties when the 2 hands were close together (in that case a touch on the inside grabs the hour hand and a touch toward the outside grabs the minute hand, but this rule has to be remembered). The 12-hour clock allows users to set a time precisely without difficulties (The prototype allows 1-minute increments).

With the 24-hour dial scheduler (Fig. 2) (designed and implemented by Jeffrey Mitchell) only one hand needs to be moved to enter a time but the reading of 24-hour pie-shape feedback on the calendar was often found confusing because of the widespread use of the 12-hour circular scale .

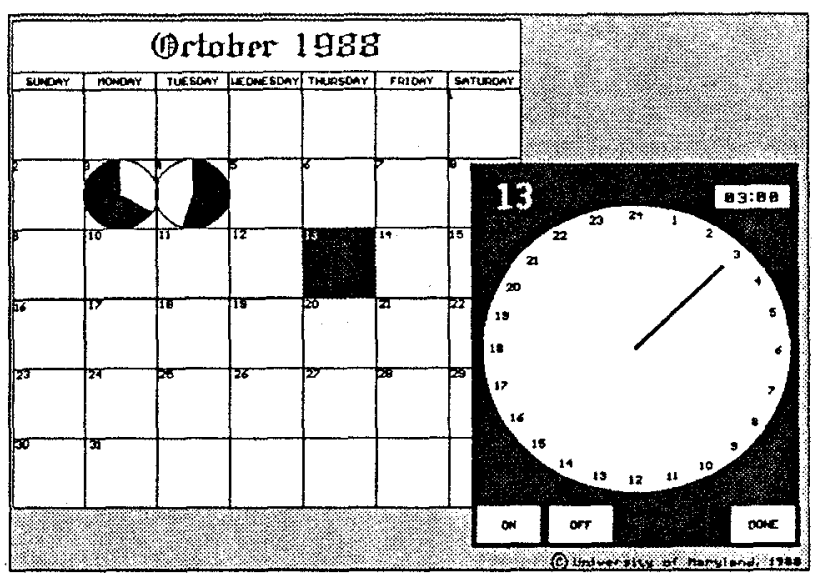

Figure 2: the 24-hour dial scheduler.

() 1988, University of Maryland

With the 24-hour linear scheduler (Fig. 3) users select dates on a calendar, a 24-hour line corresponding to the selected date appears and ON or OFF flags can be dragged onto the line.

Subjects had no difficulties using this scheduler which has many advantages: clear feedback about the duration of the event is visible on the line and the calendar, several events can be shown on the line and multiple other types of flags are possible to accommodate special needs of some devices. No AM/PM confusion was observed and users made many comments about the information that could potentially be displayed on the line (daylight, forecast, etc.).

Choosing to display a full 24-hour scale on the line limits the precision of the time selection. In the prototype only 10 minute increments were made accessible to ensure easy selection but many techniques can be used to compensate for this possible limitation (simple stabilization of the touchscreen [which was not included in the prototype shown in the videotape], scale stretching or scrolling, tuning buttons, etc.)

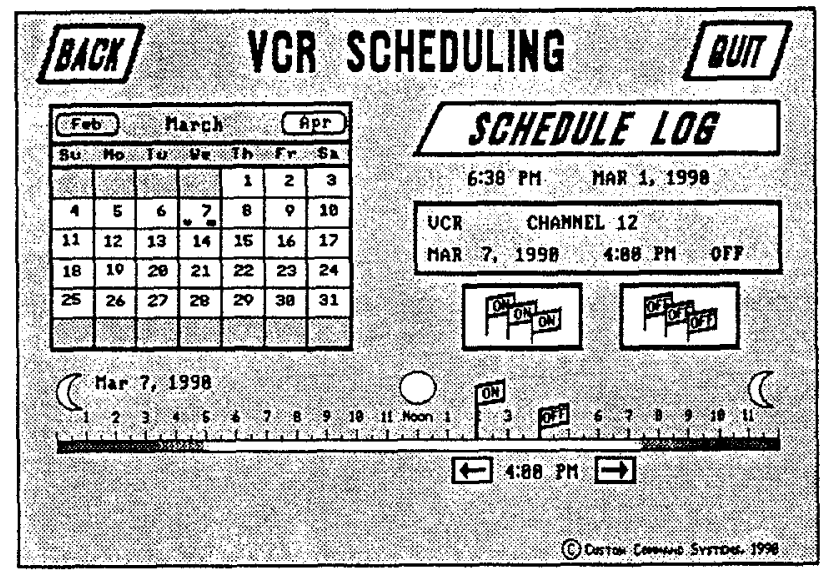

Figure 3: the 24-hour line scheduler, as it is now implemented in Custom Command system.

(c) 1988-90, Custom Command Systems and UMd

The overall result of the usability study was that each version had its strengths and weaknesses but that all three were usable by novice users. Each version had its proponents but the reviewers' feedback (visitors of the lab, customers, etc.) as well as the flexibility to add functions favors the 24-hour linear version. This version ultimately was selected to be included in Custom Command's system [3]. It is now in use in several homes. The study has since been extended to the scheduling of periodic and nonperiodic repetitive scheduling and we hope to have the opportunity to evaluate the use of the scheduler in the home. This work also allowed to explore the use of the touchscreen and demonstrate its approppriateness for applications outside the traditional button selection where it was traditionnaly confined.

\section{REFERENCES}

1. Sears, A., Plaisant, C. and Shneiderman, B. (June 1990), A new era for touchscreens: High precision, dragging icons and refined feedback. To appear in Hartson, R. and Hix, D. ed., Advances in HumanComputer Interaction, Vol.3, Ablex Publ., NJ.

2. Plaisant, C., and Shneiderman, B. (Nov. 1989), Scheduling ON-OFF home control devices: Design issues and usability evaluation of four touchscreen interfaces, to appear in the Journal of Man-Machine Studies

3. Plaisant, C. , Shneiderman, B., Battaglia, J., (Nov. 1990). Scheduling home-control devices: A case study of the transition from the research project to a product. Human-Factors in Practice (Dec. 90), Computer Systems Technical Group, Human-Factor Society, Santa-Monica, CA.

Note: The video was first presented at Interact' 90 . 